\title{
Effects of metal loading and magnetic field strength on alignment of noncrystalline block copolymers doped with metal complexes
}

\author{
Hiroaki Wakayama • Hirotaka Yonekura • \\ Masashi Harada
}

Received: 11 March 2014 / Accepted: 19 May 2014 / Published online: 10 June 2014

(C) The Author(s) 2014. This article is published with open access at Springerlink.com

\begin{abstract}
Control of the orientation of block copolymers in self-assembled nanostructures is important for their applications in organic semiconductors, lithographic nanopatterning, separation membranes, and nanofabrication templates. We recently reported that addition of magnetically sensitive metal complexes to block copolymers can be used to align the block copolymers under the influence of a magnetic field. In the present study, we investigated the mechanism of magnetic alignment of block copolymers doped with metal complexes. Specifically, we used small-angle X-ray scattering analysis to evaluate the effects the metal complex molar ratio and the strength of the applied magnetic field have on magnetic alignment in block copolymer-metal composites. Two Fe precursors, tricarbonyl(cyclooctatetraene) iron and acetylacetonate iron(III), and one Pt precursor, platinum dimethylcyclooctadiene, were selectively introduced into separate polymer blocks of a block copolymer, polystyrene-block-poly(2-vinylpyridine) (PSP2VP, $102 \mathrm{k} / 97 \mathrm{k}$ ) or polystyrene-block-poly(4-vinylpyridine) (PS-P4VP, $40 \mathrm{k} / 5.6 \mathrm{k}$ ), and the resulting films were annealed in a magnetic field. We found that magnetic alignment of the block copolymers was enhanced by high metal complex molar ratios and high magnetic field strength. The lamellar structures of the self-assembled PS-P2VP(102 k/97 k) composites were disturbed when the amounts of the metal complexes were increased, and magnetic alignment of the lamellar structures was enhanced when the strength of the applied magnetic field was increased. Magnetic alignment induced shrinkage of the cylindrical structures of the selfassembled PS-P4VP(40 k/5.6 k) composites with high metal complex ratios.
\end{abstract}

H. Wakayama $(\bowtie) \cdot \mathrm{H}$. Yonekura $\cdot$ M. Harada

Toyota Central R\&D Laboratories, Inc., Nagakute,

Aichi 480-1192, Japan

e-mail: wakayama@mosk.tytlabs.co.jp
Keywords Self-assembly $\cdot$ Block copolymers $\cdot$ Magnetic alignment $\cdot$ Nanomaterials

\section{Introduction}

Block copolymers are attracting much attention because of their ability to self-assemble into periodically ordered nanostructures. Control of the orientation of the block copolymers in such nanostructures is important for their applications in organic semiconductors [1-3], lithographic nanopatterning [4-6], separation membranes [7-9], and nanofabrication templates [10-12]. Many studies have focused on orienting block copolymers by applying stress [13-16], electric fields [17-19], and magnetic fields [20-24]. In the latter case, selfassembly of block copolymers that exhibit anisotropic magnetic susceptibility, such as liquid-crystalline block copolymers, is directed by application of a magnetic field; alignment of the block copolymers is achieved by means of orientation of the liquid-crystal mesogens. However, this approach is limited to crystalline block copolymers with anisotropy.

To expand this methodology to other types of block copolymers, including noncrystalline block copolymers, investigators have introduced magnetically sensitive additives into a specific polymer block of block copolymers. For example, introduction of a ligand containing a biphenyl moiety that interacts with the poly(acrylic acid) block of a poly(styreneblock-acrylic acid) block copolymer by hydrogen bonding has been shown to impart magnetic susceptibility, which in turn facilitates magnetic alignment of the block copolymer-ligand composite [25]. However, this method is limited by the need for a specific interaction (i.e., hydrogen bonding) between the block copolymer and the ligand.

Recently we reported a synthetic route to inorganic nanoheterostructures by templating block copolymer 
self-assembly [26]. Metal complexes are introduced into a specific copolymer block, and subsequent removal of the block copolymers by pyrolysis produces self-assembled inorganic spheres, cylinders, or layers in a matrix. In addition, the morphology of the block copolymer-metal complex composite can be transformed (from spheres to cylinders) by application of a magnetic field. By means of this method, many kinds of ligands can be introduced into noncrystalline polymer blocks, and in particular magnetic susceptibility can be imparted to many kinds of block copolymers, which can then be magnetically aligned. The elucidation of the orientation mechanism in block copolymer-metal complex composites leads to the improvement in the material properties of the block copolymer composites as well as the inorganic nanohetero materials produced after the pyrolytic removal of the block copolymer.

Here, we describe in detail the mechanism of magnetic alignment of block copolymers doped with metal complexes; specifically, we describe the effects of the metal complex molar ratio and the strength of the applied magnetic field on magnetic alignment.

\section{Experimental section}

Preparation of block copolymer composites

Tricarbonyl(cyclooctatetraene) iron $\left(\mathrm{CtFe}(\mathrm{CO})_{3},>96 \%\right.$, Tokyo Chemical Industry), dimethyl(1,5-cyclooctadiene) platinum(II) ( $\mathrm{PtMe}_{2} \mathrm{COD}, 99 \%$, Wako Pure Chemical Industries), and acetylacetonate iron(III) (Fe(acac) $3,99 \%$, Wako) were dissolved in a $0.5 \mathrm{wt} . \%$ solution of polystyreneblock-poly(2-vinylpyridine) (PS-P2VP, $M_{\mathrm{n}}{ }^{\mathrm{PS}}=102 \mathrm{~kg} \mathrm{~mol}^{-1}$, $M_{\mathrm{n}}^{\mathrm{P} 2 \mathrm{VP}}=97 \mathrm{~kg} \mathrm{~mol}^{-1}$, polydispersity index $=1.12$, Polymer Source) in toluene ( $>99.5 \%$, Wako). Several solutions were prepared, and in each solution the $\mathrm{CtFe}(\mathrm{CO})_{3} /$ styrene, $\mathrm{PtMe}_{2} \mathrm{COD} /$ vinylpyridine, and $\mathrm{Fe}(\mathrm{acac})_{3} /$ vinylpyridine molar ratios were the same, ranging from 0 to 1.0. After stirring for $3 \mathrm{~h}$, each solution was sprayed onto a Kapton film, and the samples were annealed at $453 \mathrm{~K}$ for $6 \mathrm{~h}$ in a magnetic field $(0$ $10 \mathrm{~T}$ ) applied parallel to the film. In some of the experiments, polystyrene-block-poly-(4-vinylpyridine) (PS-P4VP, $M_{\mathrm{n}}{ }^{\mathrm{PS}}=$ $40 \mathrm{~kg} \mathrm{~mol}^{-1}, M_{\mathrm{n}}^{\mathrm{P} 4 \mathrm{VP}}=5.6 \mathrm{~kg} \mathrm{~mol}^{-1}$, polydispersity index = 1.10 , Polymer Source) was used in place of the PSP4VP(102 k/97 k).

Characterization of block copolymer composites

Scanning transmission electron microscopy of the resulting composites was conducted with a high-resolution transmission electron microscope (JEM-2010FEF, JEOL) operating at $200 \mathrm{keV}$. In addition, small-angle X-ray scattering (SAXS) measurements were performed on the BL33XU beamline at SPring-8 (Hyogo, Japan). The incident X-rays were applied normal to the samples for through-view images and parallel to the samples (and in the direction of the applied magnetic field) for cross-view images.

\section{Results and discussion}

Scanning transmission electron microscopy

Scanning transmission electron microscopy images of samples that were prepared with PS-P2VP(102 k/97 k) and PS$\mathrm{P} 4 \mathrm{VP}(40 \mathrm{k} / 5.6 \mathrm{k})$ and metal complexes at molar ratios of 1.0 and that were annealed in a $10 \mathrm{~T}$ magnetic field showed lamellar and cylindrical structures, respectively, that were almost exactly parallel to the direction of the applied magnetic field (Fig. 1).

Effects of metal complex molar ratio and magnetic field strength on block copolymer composite structure

Through-view images obtained by applying X-rays normal to the samples prepared with PS-P2VP(102 k/97 k) and PSP4VP(40 k/5.6 k) (metal complex molar ratio, 1.0; magnetic field, $10 \mathrm{~T}$ ) showed isotropic two-dimensional patterns (Fig. 2). Figure 3 shows the one-dimensional SAXS profiles obtained from the circular integral of the scattering intensity at the distance from the scattering center. In the SAXS pattern of a sample prepared with PS-P4VP(102 k/97 k) without metal complexes and at magnetic field strength of $10 \mathrm{~T}$, the relative positions of the 1:2:3 peaks confirmed that the sample had a lamellar structure (Fig. 3a metal complex molar ratio, 0.0; blue line). As the amounts of the metal complexes were increased, the first peaks broadened (Fig. 3a), which suggests that the lamellar structures were disturbed by introduction of the metal complexes. The first peaks become sharp as the strength of the applied magnetic field was increased
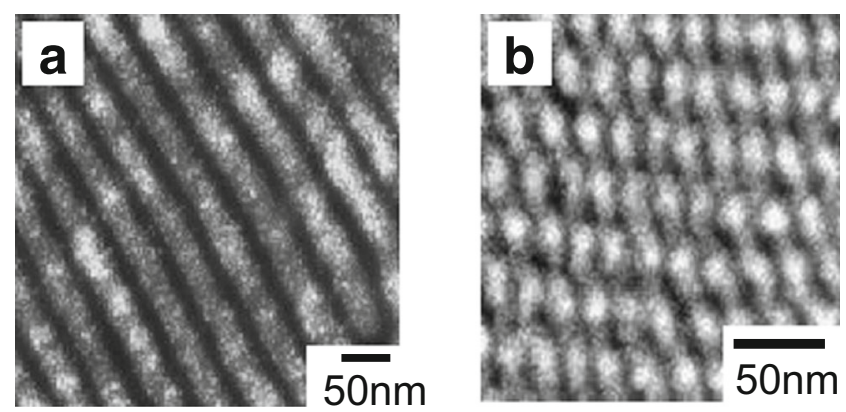

Fig. 1 Scanning transmission electron microscopy images of samples prepared from (a) PS-P2VP(102 k/97 k) and (b) PS-P4VP(40 k/5.6 k) and metal complexes (1.0 molar ratio) and annealed in a $10 \mathrm{~T}$ magnetic field 
Fig. 2 Two-dimensional scattering patterns in throughview images obtained by applying X-rays normal to samples prepared with (a) PS-P2VP(102 k/97 k) and (b) PS-P4VP(40 k/5.6 k) and metal complexes (1.0 molar ratio) and annealed in a $10 \mathrm{~T}$ magnetic field a
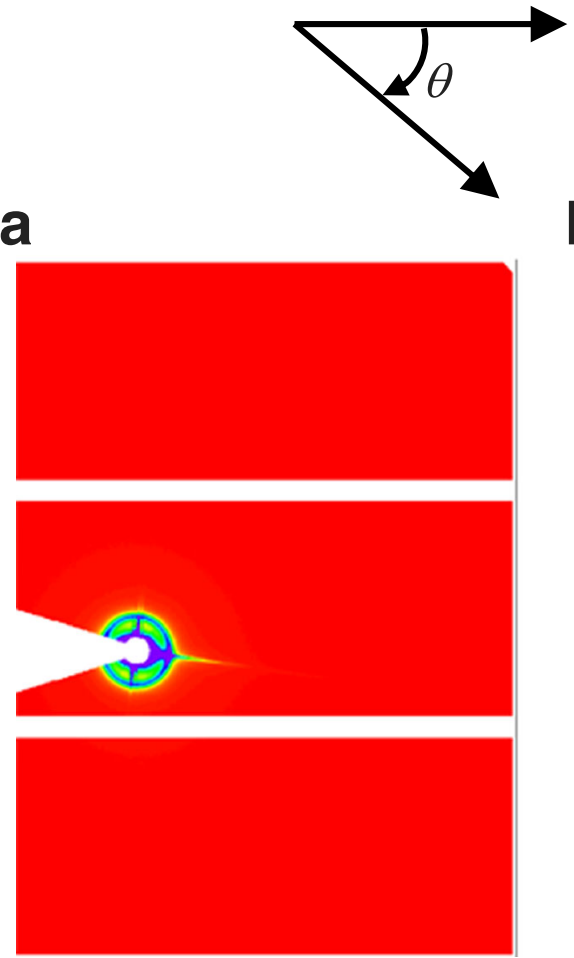

b
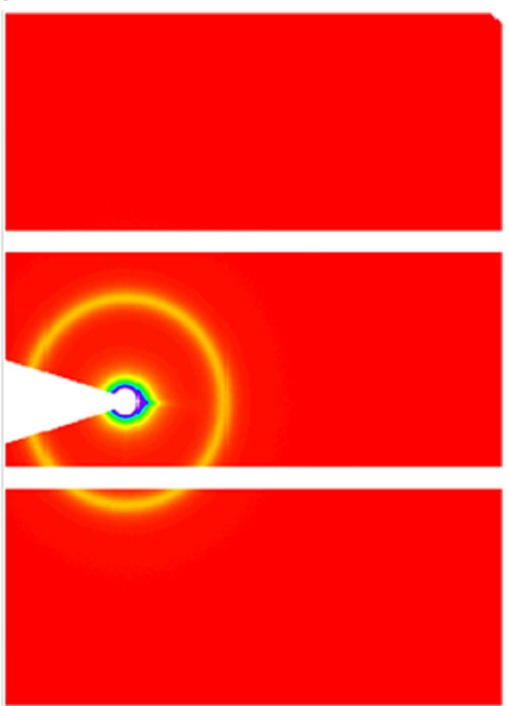

(Fig. 3b). This result is attributable to magnetic alignment of the self-assembled lamellar structures.

As was the case for the SAXS pattern of the sample prepared with PS-P2VP(102 k/97 k), in the SAXS pattern of the sample prepared with PS-P4VP(40 k/5.6 k), the first peaks broadened as the amounts of metal complexes were increased (Fig. 3c), and the first peaks become sharp as the strength of the applied magnetic field was increased (Fig. 3d), suggesting magnetic alignment of the self-assembled cylindrical structures. As shown in Fig. 3d, the peaks at relative positions of 1:
Fig. 3 Effects of metal complex molar ratio (magnetic field, $10 \mathrm{~T}$; left panels) and magnetic field strength (metal complex molar ratio, 1.0 ; right panels) on onedimensional small-angle X-ray scattering profiles obtained from the circular integral of the scattering intensity at the distance from the scattering center: $\mathbf{a}, \mathbf{b}$ PS-P2VP(102 k/97 k) and c, d PS-P4VP(40 k/5.6 k)
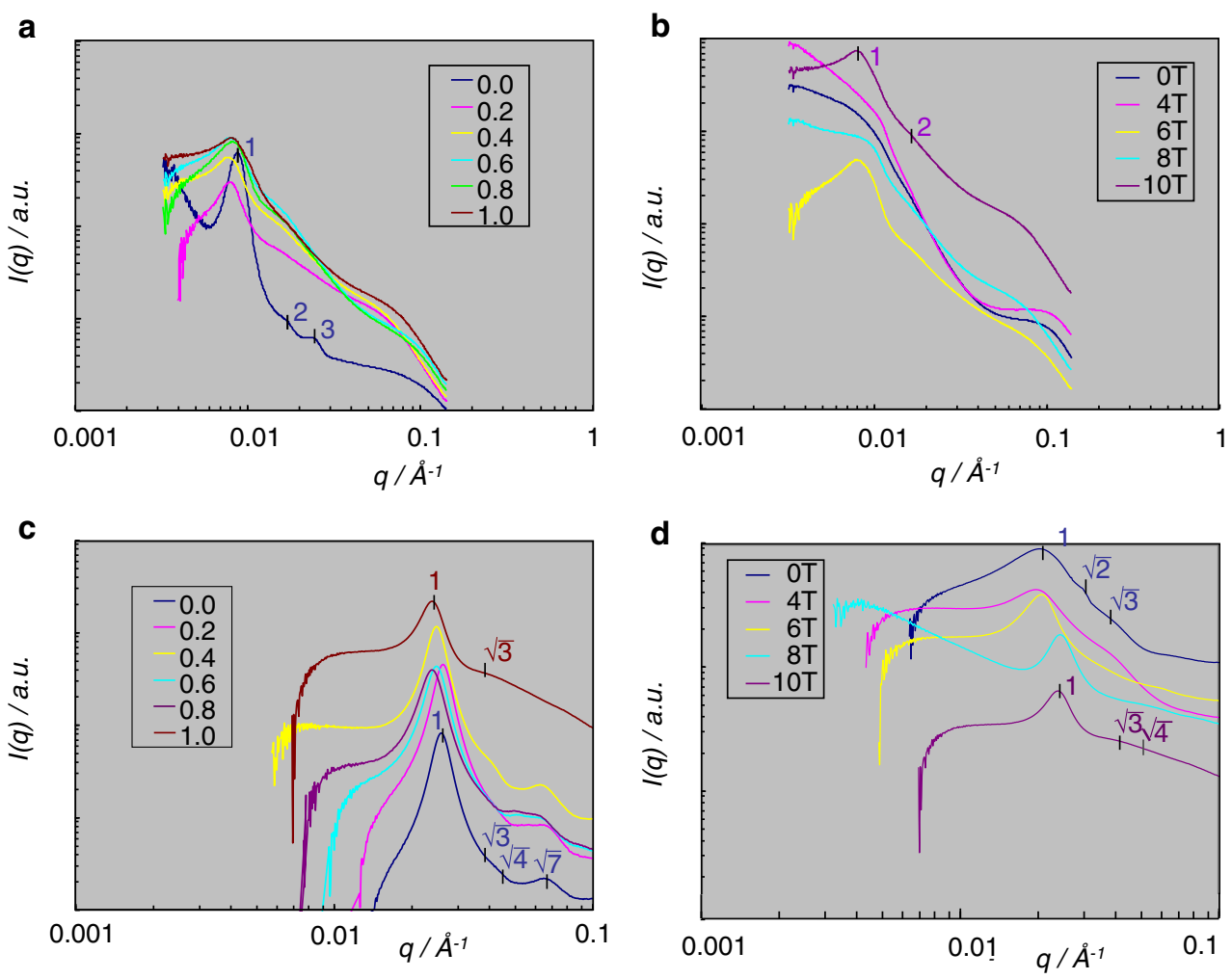
$\sqrt{2}: \sqrt{3}$ for the samples prepared with PS-P4VP(40 k/5.6 k) in the absence of a magnetic field (Fig. 3d magnetic field, $0 \mathrm{~T}$; blue line) and the peaks at relative positions of $1: \sqrt{ } 3: \sqrt{ } 4$ for the samples prepared with PS-P4VP $(40 \mathrm{k} / 5.6 \mathrm{k})$ and annealed in a high magnetic field (Fig. 3d magnetic field, $10 \mathrm{~T}$; purple line) confirmed the spherical and hexagonal cylinder structures, respectively, of these samples. That is, application of a magnetic field to the PS-P4VP(40 k/5.6 k)-metal complex composite resulted in a phase transition from spheres to hexagonal cylinders.

Effects of metal complex molar ratio and magnetic field strength on the repeat size of the block copolymer composites

In the through-view images of the block copolymer composites(Fig. 4), the repeat size of samples prepared with PS-P2VP(102 k/97 k) and PS-P4VP(40 k/5.6 k) and annealed in a $10 \mathrm{~T}$ magnetic field increased with increasing metal complex molar ratio up to a ratio of 0.6 , whereas at ratios higher than 0.6 , the repeat size decreased. This result suggests that the polymer blocks of the microphaseseparated structures were swollen by the introduction of metal complexes at molar ratios of $<0.6$ and that shrinkage was induced by magnetic alignment of the samples containing magnetically sensitive metal complexes at molar ratios of $>0.6$. As shown in Fig. 5, the repeat sizes of samples prepared with PS-P2VP(102 k/97 k) and PS$\mathrm{P} 4 \mathrm{VP}(40 \mathrm{k} / 5.6 \mathrm{k})$ decreased with increasing magnetic field strength at values of $>6 \mathrm{~T}$, suggesting magnetic alignment of the block copolymer composite structures.

Azimuthal angle dependence of peak intensity

The azimuthal angle dependence of the intensity of the first peak in the through-view images of samples prepared with PS-P2VP(102 k/97 k) and PS-P4VP(40 k/5.6 k)
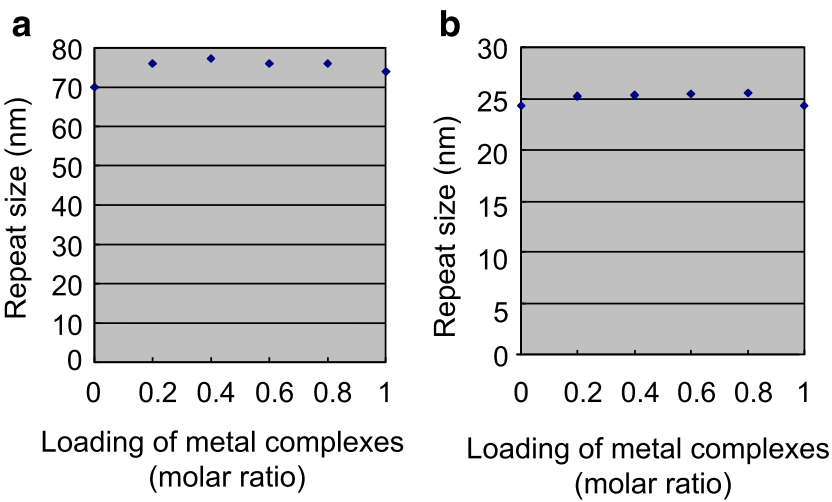

Fig. 4 Effects of metal complex molar ratio on the repeat size of block copolymer composites prepared with (a) PS-P2VP(102 k/97 k) and (b) PS-P4VP(40 k/5.6 k) annealed in a $10 \mathrm{~T}$ magnetic field
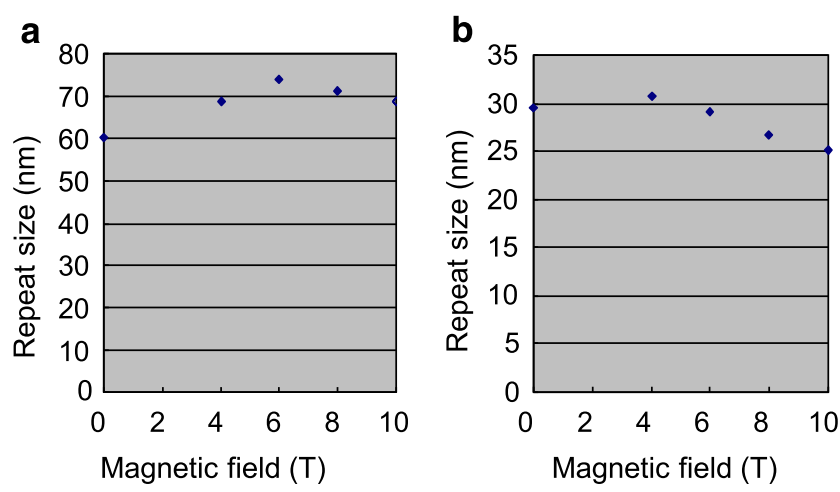

Fig. 5 Effect of magnetic field strength on the repeat size of block copolymer composites prepared from (a) PS-P2VP(102 k/97 k) and (b) PS-P4VP(40 k/5.6 k) and metal complexes (1.0 molar ratio)

(metal complex molar ratio, 1.0; magnetic field strength, $10 \mathrm{~T}$ ) is shown in Fig. 6; the intensity of the first peak was almost independent of the azimuthal angle; no clear orientation induced by the magnetic field was observed. The results for the cross-view images are shown in Fig. 7 at the various metal complex molar ratios and magnetic field strengths. The intensities of the first peaks were maximal in the normal direction $\left(0^{\circ}\right.$ and $\left.180^{\circ}\right)$ for the samples prepared with PS-P2VP(102 k/97 k) and PS$\mathrm{P} 4 \mathrm{VP}(40 \mathrm{k} / 5.6 \mathrm{k})$. This suggests that the self-assembled structures were oriented parallel to the film samples. For both PS-P2VP(102 k/97 k) and PS-P4VP(40 k/5.6 k), the peak intensity increased as the strength of the applied magnetic field was increased (Fig. 7a, c) and as the amounts of metal complexes were increased (Fig. 7b, d). Samples prepared with PS-P2VP(102 k/97 k) (metal complex molar ratio, 0.0; magnetic field strength, $10 \mathrm{~T}) \mathrm{ex}-$ hibited small peaks at around $90^{\circ}$ and $270^{\circ}$ (Fig. $7 \mathrm{~b}$ blue line), which were attributed to disturbance of the orientation of the lamellar structures. In contrast, broad peaks at $50-150^{\circ}$ and $230-330^{\circ}$ were observed for the samples that were prepared with PS-P4VP(40 k/5.6 k) annealed at high magnetic field strength (Fig. $7 \mathrm{c}$ magnetic field strength, $10 \mathrm{~T}$ : purple line) or the samples that were
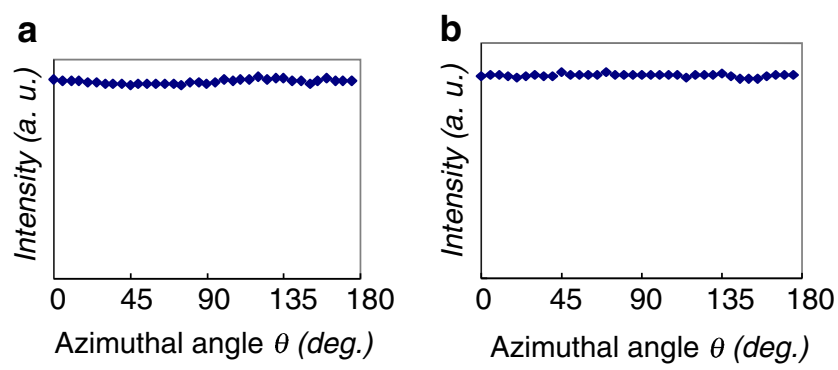

Fig. 6 Azimuthal angle dependence of the intensity of the first peak in the through-view images of composites prepared with (a) PSP2VP(102 k/97 k) and (b) PS-P4VP(40 k/5.6 k) and metal complexes (1.0 molar ratio) and annealed in a $10 \mathrm{~T}$ magnetic field 
Fig. 7 Effects of magnetic field strength (metal complex molar ratio, 1.0; left panels) and metal complex molar ratio (magnetic field, $10 \mathrm{~T}$; right panels) on the azimuthal angle dependence of the intensity of the first peak in the cross-view images of composites prepared with (a, b) PS-

P2VP(102 k/97 k) and (c, d) PS$\mathrm{P} 4 \mathrm{VP}(40 \mathrm{k} / 5.6 \mathrm{k})$
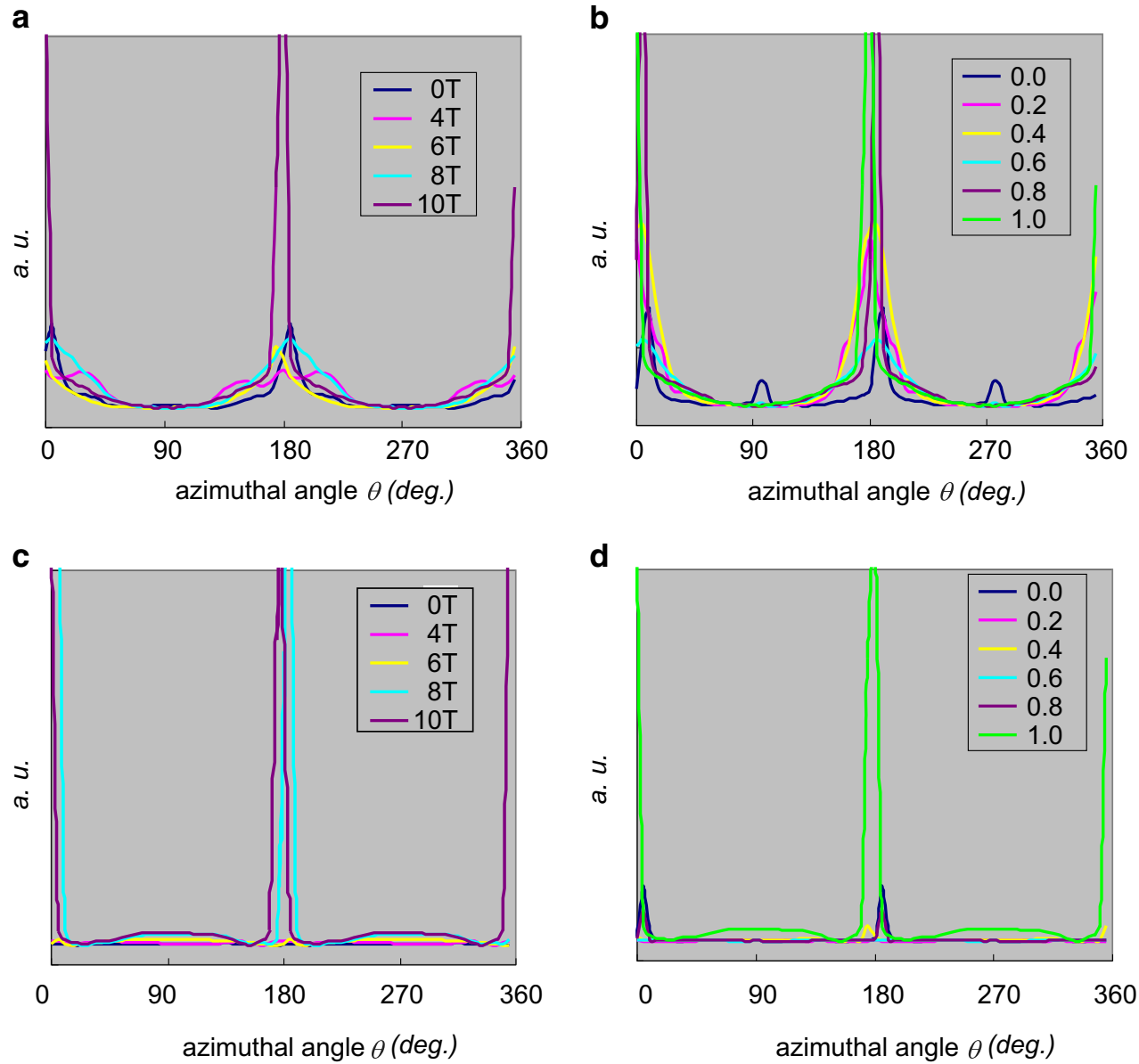

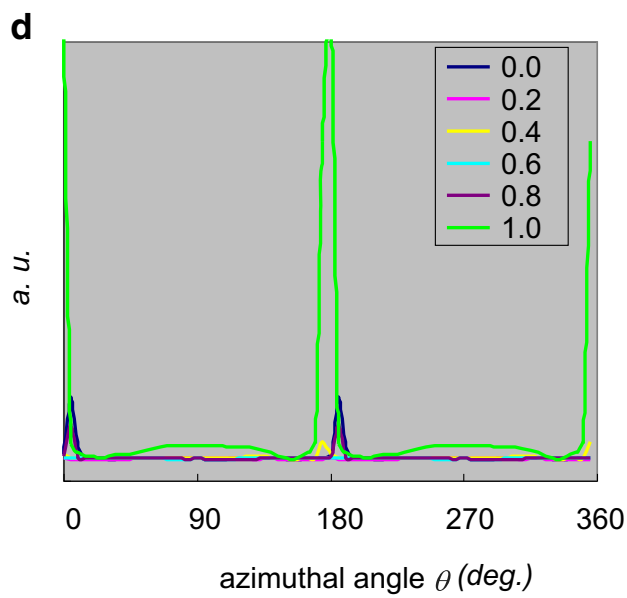

prepared with PS-P4VP(40 k/5.6 k) and a large amount of metal complexes (Fig. 7d metal complex molar ratio, 1.0: light green line). These broad peaks were due to the high orientation of the hexagonally packed cylindrical structures [27]. The highly oriented cylindrical structures were

a
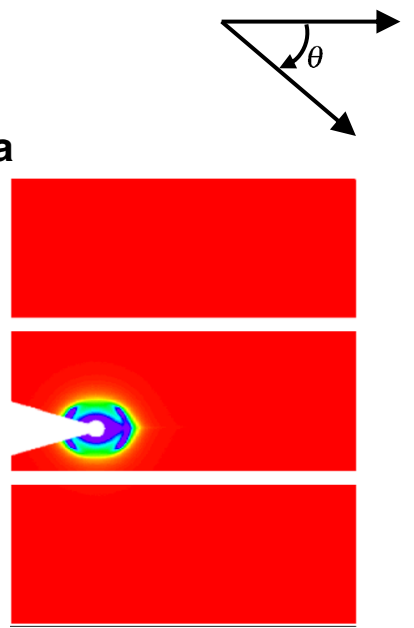

b
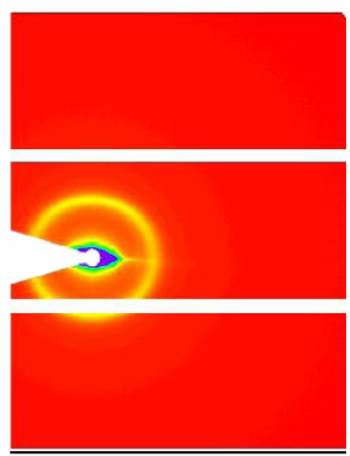

Fig. 8 Two-dimensional scattering patterns in cross-view images obtained by applying X-rays parallel to composites prepared with (a) PSP2VP(102 k/97 k) and (b) PS-P4VP(40 k/5.6 k) and metal complexes (1.0 molar ratio) and annealed in a $4 \mathrm{~T}$ magnetic field induced by a high loading of metal complexes and by annealing in a high-strength magnetic field.
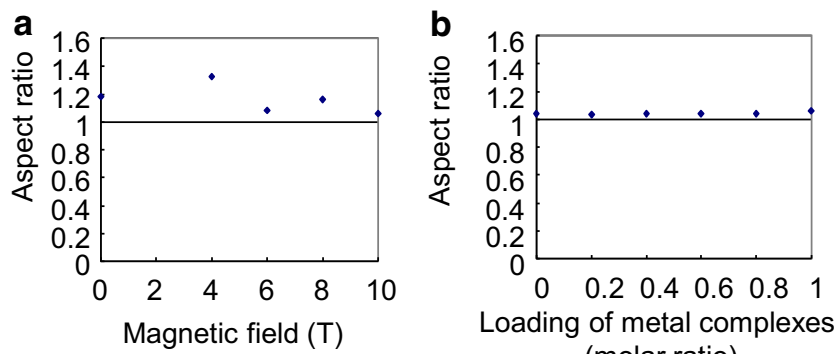

Loading of metal complexes (molar ratio)
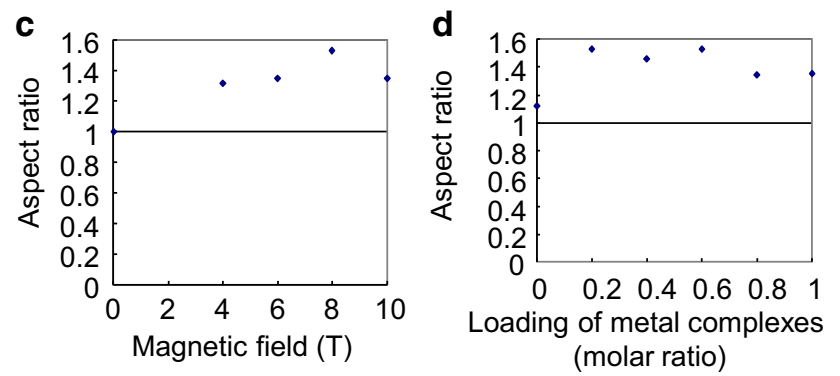

Fig. 9 Effects of magnetic field strength (metal complex molar ratio, 1.0; left panels) and metal complex molar ratio (magnetic field, $10 \mathrm{~T}$; right panels) on the aspect ratio of the scattering ring: a, b PS-P4VP(40 k/5.6 k) and c, d PS-P2VP(102 k/97 k) 
Ellipse aspect ratio of two-dimensional SAXS pattern

For the PS-P2VP(102 k/97 k) and PS-P4VP(40 k/5.6 k) samples prepared at a metal complex molar ratio of 1.0 and a magnetic field strength of $10 \mathrm{~T}$, the intensity of the first peak in the through-view images were almost independent of the azimuthal angle (Fig. 6), and the aspect ratio of the scattering ring in the two-dimensional SAXS pattern was almost 1 (Fig. 2), suggesting isotopic scattering. Figure 8 shows the two-dimensional cross-view SAXS patterns for the PSP2VP(102 k/97 k) and PS-P4VP(40 k/5.6 k) samples prepared at a metal complex molar ratio of 1.0 and a magnetic field strength of $4 \mathrm{~T}$. The fact that the scattering ring for the sample prepared with PS-P4VP(40 k/5.6 k) had an oval shape extending in the equatorial direction (Fig. $8 \mathrm{~b}$ ) indicates that the repeat size of the cylindrical structure was smaller in the normal direction of the film samples than in the parallel direction. As the magnetic field strength was increased, the aspect ratio of the scattering ring approached 1 (Fig. 9a), which suggests that the cylindrical structures of PS$\operatorname{P} 4 \mathrm{VP}(40 \mathrm{k} / 5.6 \mathrm{k})$ were aligned by the application of the magnetic field and that the alignment reduced distortion of the hexagonally packed structures [28]. For the lamellar structures from PS-P2VP(102 k/97 k), it is considerable that anisotropy of the vertical and horizontal direction occurred in the polymer block, such as polymer density, induced by the application of magnetic field.

\section{Conclusions}

To investigate the mechanism of magnetic alignment of block copolymers, we examined the effects of metal complex molar ratio and magnetic field strength on magnetic alignment in self-assembled block copolymer composites. We found that alignment was enhanced by high metal complex molar ratio and high magnetic field strength. As the amounts of metal complexes in the composites were increased, the lamellar structures were disturbed. The magnetic alignment of the lamellar structures was enhanced by increasing the strength of the applied magnetic field. For cylindrical structures, shrinkage was induced by magnetic alignment of samples prepared at high metal complex molar ratios. Our results can be expected to contribute to the extension of magnetic alignment methods to various kinds of block copolymers including noncrystalline block copolymers. The control of the orientation in block copolymer-metal complex composites leads to the improvement in the material properties of the block copolymer composites as well as the inorganic nanohetero materials produced after the pyrolytic removal of the block copolymer. Our approach is simple and widely applicable for the synthesis of heterogeneous nanostructured materials with controlled oriented structures.

Acknowledgments The SAXS experiments were performed on the BL33XU beamline at SPring-8 with the approval of the Japan Synchrotron Radiation Research Institute (JASRI; proposal no. 2013B7003). We acknowledge T. Matsunaga for analysis of SAXS data.

Open Access This article is distributed under the terms of the Creative Commons Attribution License which permits any use, distribution, and reproduction in any medium, provided the original author(s) and the source are credited.

\section{References}

1. Zorn M, Zentel R (2008) Liquid crystalline orientation of semiconducting nanorods in a semiconducting matrix. Macromol Rapid Commun 29:922-927

2. Tao Y, Ma B, Segalman RA (2008) Self-assembly of rod-coil block copolymers and their application in electroluminescent devices. Macromolecules 41:7152-7159

3. Lee YJ, Kim SH, Yang H, Jang M, Hwang SS, Lee HS, Baek KY (2011) Vertical conducting nanodomains self-assembled from poly(3-hexyl thiophene)-based diblock copolymer thin films. J Phys Chem C 115:4228-4234

4. Jung YS, Ross CA (2007) Orientation-controlled self-assembled nanolithography using a polystyrene-polydimethylsiloxane block copolymer. Nano Lett 7:2046-2050

5. Ramanathan M, Nettleton E, Darling SB (2009) Simple orientational control over cylindrical organic-inorganic block copolymer domains for etch mask applications. Thin Solid Films 517:4474-4478

6. Xie N, Li WH, Qiu F, Shi AC (2013) New strategy of nanolithography via controlled block copolymer self-assembly. Soft Matter 9:536-542

7. Rzayev J, Hillmyer MA (2005) Nanochannel array plastics with tailored surface chemistry. J Am Chem Soc 127:13373-13379

8. Barbi V, Funari SS, Gehrke R, Scharnagl N, Stribeck N (2003) SAXS and the gas transport in polyether-block-polyamide copolymer membranes. Macromolecules 36:749-758

9. Armstrong S, Freeman B, Hiltner A, Baer E (2012) Gas permeability of melt-processed poly(ether block amide) copolymers and the effects of orientation. Polymer 53:1383-1392

10. Li M, Ober CK (2006) Block copolymer patterns and templates. Mater Today 9:30-39

11. Lee JI, Cho SH, Park SM, Kim JK, Kim JK, Yu JW, Kim YC, Russell TP (2008) Highly aligned ultrahigh density arrays of conducting polymer nanorods using block copolymer templates. Nano Lett 8: 2315-2320

12. Chai J, Buriak JM (2008) Using cylindrical domains of block copolymers to self-assemble and align metallic nanowires. ACS Nano 2: 489-501

13. Laiho A, Hiekkataipale P, Ruokolainen J, Ikkala O (2009) Directing the smectic layer orientation by shear flow in hierarchical lamellarwithin-lamellar liquid crystalline diblock copolymers. Macromol Chem Phys 210:1218-1223

14. Koppi KA, Tirrell M, Bates FS, Almdal K, Colby RH (1992) Lamellae orientation in dynamically sheared diblock copolymer melts. J Phys II 2:1941-1959

15. Honeker CC, Thomas EL (1996) Impact of morphological orientation in determining mechanical properties in triblock copolymer systems. Chem Mater 8:1702-1714 
16. Mai B, Li Z, Liu R, Feng S, Wu Q, Liang G, Gao H, Zhu F (2013) Confined crystallization of core-forming blocks in nanoscale self-assembled micelles of poly( $\varepsilon$-caprolactone)-bpoly(ethylene oxide) in aqueous solution. J Polym Res. doi: 10.1007/s10965-013-0299-x

17. Thurn-Albrecht T, DeRouchey J, Russell TP, Kolb R (2002) Pathways toward electric field induced alignment of block copolymers. Macromolecules 35:8106-8110

18. Elhadj S, Woody JW, Niu VS, Saraf RF (2003) Orientation of selfassembled block copolymer cylinders perpendicular to electric field in mesoscale film. Appl Phys Lett 82:871-873

19. Tsori Y, Andelman D (2002) Thin film diblock copolymers in electric field: transition from perpendicular to parallel lamellae. Macromolecules 35:5161-5170

20. Tao YF, Zohar H, Olsen BD, Segalman RA (2007) Hierarchical nanostructure control in rod-coil block copolymers with magnetic fields. Nano Lett 7:2742-2746

21. Gopinadhan M, Majewski PW, Osuji CO (2010) Facile alignment of amorphous poly(ethylene oxide) microdomains in a liquid crystalline block copolymer using magnetic fields: toward ordered electrolyte membranes. Macromolecules 43:3286-3293

22. Grigorova T, Pispas S, Hadjichristidis N, Thurn-Albrecht $T$ (2005) Magnetic field induced orientation in diblock copolymers with one crystallizable block. Macromolecules 38:7430-7433

23. Tomikawa N, Lu ZB, Itoh T, Imrie CT, Adachi M, Tokita M, Watanabe J (2005) Orientation of microphase-segregated cylinders in liquid crystalline diblock copolymer by magnetic field. Jpn J Appl Phys 2(44):L711-L714

24. Tran H, Gopinadhan M, Majewski PW, Shade R, Steffes V, Osuji C, Campos LM (2013) Monoliths of semiconducting block copolymers by magnetic alignment. ACS Nano 7:5514-5521

25. Gopinadhan M, Majewski PW, Beach ES, Osuji CO (2012) Magnetic field alignment of a diblock copolymer using a supramolecular route. ACS Macro Lett 1:184-189

26. Wakayama H, Yonekura H, Kawai Y (2013) Threedimensional periodically ordered nanohetero metallic materials from self-assembled block copolymer composites. ACS Macro Lett 2:284-287

27. Sakurai S (2008) Progress in control of microdomain orientation in block copolymers-efficiencies of various external fields. Polymer 49: 2781-2796

28. Sakurai S, Momii T, Taie K, Shibayama M, Nomura S, Hashimoto T (1993) Morphology transition from cylindrical to lamellar microdoains of block copolymers. Macromolecules $23: 485-491$ 